\title{
Stability of the 3-torus solution in a ring of coupled Duffing oscillators
}

\author{
L. Borkowski ${ }^{1}$ and A. Stefanski ${ }^{2, a}$ \\ ${ }^{1}$ Department of Strength of Materials, Lodz University of Technology, \\ ul. Stefanowskiego 1/15 Lodz, Poland \\ 2 Division of Dynamics, Lodz University of Technology, ul. Stefanowskiego 1/15 Lodz, \\ Poland
}

Received 8 December 2019 / Accepted 8 June 2020

Published online 28 September 2020

\begin{abstract}
The dynamics of the ring of unidirectionally coupled singlewell Duffing oscillators is analyzed in numerical simulation for identical nodal oscillators. The research is concentrated on the existence of the stable 3D torus attractor in this system. It is shown that 3-frequency quasi-periodicity can be robustly stable in wide range of parameters of the system under consideration. As an explanation of this stability, the conjecture on the coexistence and superposition of two independent effects characterized with irrational frequencies, i.e., the classical Newhouse, Ruelle and Takens scenario and rotating wave flow, is formulated.
\end{abstract}

\section{Introduction}

One of typical routes to chaotic motion of nonlinear dynamical systems is a transition via quasi-periodic solutions appearing as a result of consecutive Hopf bifurcations introducing new mode with incommensurate frequency. First time such a scenario of dimensionally increasing quasiperiodicity has been proposed by Landau [1] and Hopf [2] as an explanation of transition to the turbulence. However, later work by Newhouse, Ruelle and Takens [3] had demonstrated that just after third step of this bifurcational scenario there appear chaotic strange attractor as an effect of arbitrarily small perturbation of the 3D torus (NRT scenario). On the other hand, other researchers have shown numerical [4] and experimental results that confirm the possibility of the stable 3D [5-10] or even 4D [11] torus existence. The $4 \mathrm{D}$ torus has been also detected for three coupled circle maps [12]. The NRT theorem has been challenged by Grebogi et al. a few years after its publication $[13,14]$. They have performed a numerical experiment which confirmed that smooth nonlinear perturbations do not destroy the stability of the three-frequency quasi-periodicity, what is important from physical applications point of view. The analysis of the 3D-torus stability has been continued in the last decade of the 20th century and in the current century [15-33]. Here, especially noteworthy are works by Feudel et al. [20,21] and Anischenko et al. [22]. Their justification for this phenomenon refers to the symmetry of the analyzed system. The high-dimensional quasiperiodicity (i.e. $N \geq 3$ ) has been reported also

a e-mail: andrzej.stefanski@p.lodz.pl 
in sets of coupled systems $[12,15-19,23-29]$ and self or externally driven oscillators [30-33]. Other notable cases of such solutions (four- and five-frequency torus) have been recently demonstrated in systems of chains or globally coupled phase oscillators with frequency detuning $[34,35]$ and in system of linked delayed logistic maps [36].

In this paper the subject of the research is a ring of unidirectionally coupled single-well Duffing oscillators. The existence of robustly stable 3D torus attractor in this system, which usually appears as a result of the transition from periodic (PRW) to chaotic rotating waves (CRW), is analyzed [27-29]. This article refers directly to our previous publication on the experimental observation of the 3-frequency quasiperiodic solution in a ring of seven coupled Duffing oscillators [9] and indirectly to other related works [37-39].

At present, there are several theories regarding the problem of disagreement with NRT theorem in rings of coupled oscillators. One of them refers to a property of spatio-temporal symmetry of the invariant ring of identical oscillators under the cyclic group $[17,27]$. Other one is connected with the rotational symmetry and the suppression of lockings in modulated rotating waves [40]. Another yet is based on some discrepancy in frequencies of the torus [29] diminishing an effect of frequency resonance which is destroying for the torus.

Our experimental and numerical study proves that this solution is kept on, in spite of some symmetry breaking caused by parameters mismatch [9]. Thus, this paper focuses on the study of identical coupled oscillators. Main purpose of this study is to identify the mechanism of the 3D torus stability. We propose, as an explanation of this phenomenon, the conjecture on the coexistence and superposition of two independent effects characterized with irrational frequencies, i.e., the classical NRT scenario and rotating wave flow.

\section{Investigated system}

In general, the dynamics of a closed ring of $N$ unidirectionally coupled identical oscillators can be described in the block form:

$$
\dot{\mathbf{x}}=\mathbf{F}(\mathbf{x})+[\sigma(\mathbf{G}-\mathbf{I}) \otimes H] \mathbf{x}
$$

where $\mathbf{x}=\left(x_{1}, \ldots, x_{N}\right) T, x_{j} \in \mathbf{R}^{m}(j=1,2, \ldots, N), \mathbf{F}(\boldsymbol{x})=\operatorname{diag}\left[\mathbf{f}\left(\mathbf{x}_{1}\right), \ldots, f\left(x_{N}\right)\right]$ is a block matrix of uncoupled node systems, $\mathbf{G}$ is the $N \times N$ connectivity matrix representing the topology of connections between the ring nodes, $\mathbf{I}$ is the $N \times N$ unit matrix, $H: \mathbf{R}^{m} \rightarrow \mathbf{R}^{m}$ is an output function of each oscillator's variables that is used in the coupling (it is the same for all nodes), $\sigma$ is overall coupling coefficient and $\otimes$ is a direct (Kronecker) product of two matrices. For the unidirectional ring the matrix G has a following structure

$$
\mathbf{G}=\left[\begin{array}{ccccc}
0 & 0 & 0 & \cdots & 1 \\
1 & 0 & 0 & \cdots & 0 \\
\vdots & \vdots & \vdots & \vdots & \vdots \\
0 & \cdots & 1 & 0 & 0 \\
0 & \cdots & 0 & 1 & 0
\end{array}\right]
$$

As a node system we take autonomous single-well Duffing oscillator described by the following second order ODE:

$$
\ddot{x}+d \dot{x}+a x+b x^{3}=0
$$


where $a, b$ and $d$ are real positive parameters. Introducing the substitution $y=\dot{x}$ and assuming diffusive coupling between the oscillators according to output function

$$
H=\left(\begin{array}{ll}
0 & 0 \\
1 & 0
\end{array}\right),
$$

where $k_{j}$ is the coupling coefficient for each individual oscillator, we can describe the dynamics of each $j$ th ring node by the following pair of 1st order dimensionless ODEs:

$$
\begin{aligned}
& \dot{x}_{j}=y_{j}, \\
& \dot{y}_{j}=-a x_{j}-b x_{j}^{3}-d y_{j}+\sigma\left(x_{j-1}-x_{j}\right) .
\end{aligned}
$$

When the node oscillators are uncoupled $(\sigma=0)$, then the solution of equation (5) tends to a stable fixed point $\left(x_{j}=0, y_{j}=0\right)$ in the phase space due to a presence of damping $(d>0)$ and lack of forcing. Then we observe free damped vibrations.

\section{System analysis}

In this section, the results of numerical research of the system under consideration (Eq. (5)), are demonstrated. Particular attention is paid to the observation of threefrequency quasiperiodic solutions. Initially, our research of the system (5) was focused on the existence of rotating waves (periodic, quasi-periodic and chaotic), the stability of equilibrium (asymptotic continuous spectrum - ACS), so-called Eckhaus effect $[41,42]$ and the transition to complex dynamics for a large number of oscillators in the ring $[37,38]$. These studies have shown that the 3-frequency quasiperiodicity occurs for at least 5 oscillators in the loop $(N \geq 5)$. As a number of units increases, its existence interval in the space of coupling parameter $\sigma$ decreases, and then for $N>$ 20 practically disappears. The analysis presented here corresponds to our previous experimental and numerical (taking into account the parameters mismatch) studies [9], where a loop of seven unidirectionally coupled Duffing oscillators has been investigated. Therefore, here also seven, but identical, node systems in equation (5) with following values of parameters: $a=1.0, b=10.0, d=0.03162$, have been analyzed. These dimensionless values correspond directly to nominal coefficients of the experimental circuit [9]. The overall coupling coefficient $\sigma$ is considered as the control parameter.

\subsection{Destabilization of equilibrium and onset of rotating waves}

The investigation of the system (5), linearized in the neighborhood of the equilibrium $\mathbf{x}=[0, \ldots, 0]^{T}$, allows one to determine the value of coupling strength at which the first Hopf bifurcation initiates the rotating wave. The threshold $\sigma_{1}$ required for its occurrence can be determined by eigenvalues of the variational equation of equation (1):

$$
\delta \dot{\mathbf{x}}=[D \mathbf{F}(\mathbf{x})+\sigma(\mathbf{G}-\mathbf{I}) \otimes D H] \delta \mathbf{x}
$$

where $D \mathbf{F}$ is a diagonal block matrix of linearized Jacobi matrixes $D f\left(x_{j}\right)$ of uncoupled node systems (Eq. (5)), i.e., $D f\left(x_{j}\right)=\left(\begin{array}{cc}0 & 1 \\ -a+3 b x^{2} & d\end{array}\right)$ and $D H=H$ 


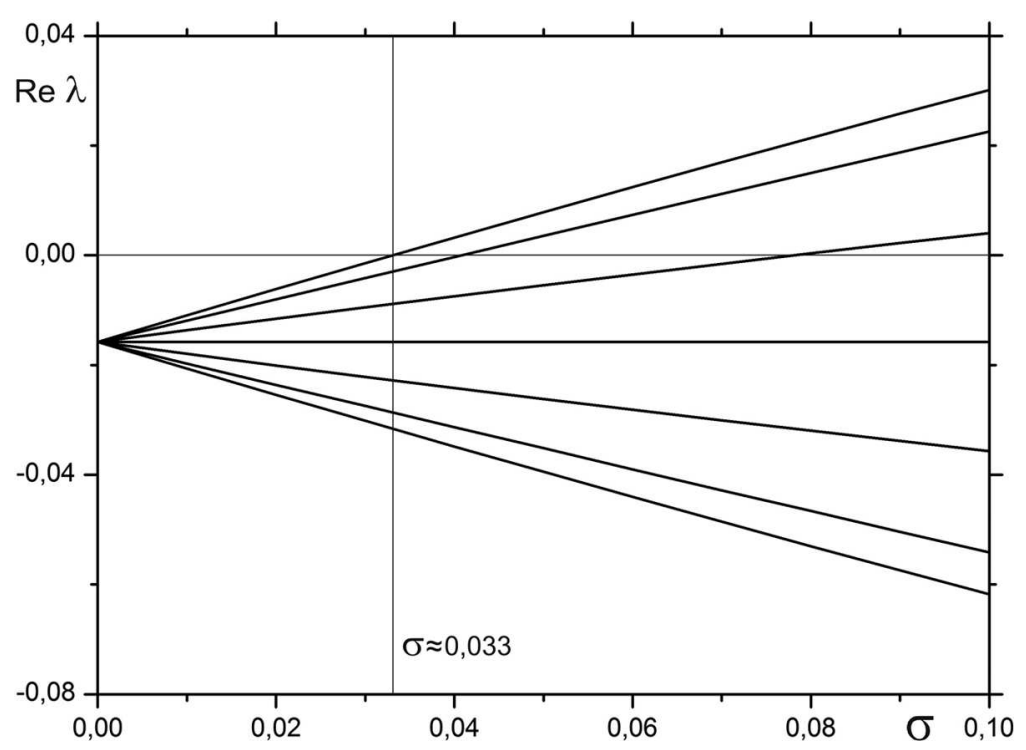

Fig. 1. Graph of the real parts of the eigenvalues Re $\lambda$ of the linearized system (6) as a function of the coupling parameter $\sigma$ for the ring of seven identical Duffing oscillators.

(Eq. (4)). Hence, substituting equation (5) into equation (6) and linearizing in the neighborhood of point $\left(x_{j}=0, y_{j}=0\right)$ we have:

$$
\begin{aligned}
& \delta \dot{x}_{j}=\delta y_{j} \\
& \delta \dot{y}_{j}=-(a+\sigma) \delta x_{j}-d \delta y_{j}+\sigma \delta x_{j-1}
\end{aligned}
$$

In the case of symmetric ring of identical items each pair of complex conjugate eigenvalues of the system (7) can be calculated according to the following general formula [37]:

$$
\lambda_{1,2}(j)=-\frac{d}{2} \pm \sqrt{\left(\frac{d}{2}\right)^{2}-a-\sigma\left(1-e^{i 2 \pi j / N}\right)} .
$$

The Hopf bifurcation activating the PRW takes place when the increase of coupling coefficient causes that the largest real part of the eigenvalue connected with $j=2$ (Eq. (8)) becomes positive at $\alpha=0.0330$, as shown in Figure 1 .

\subsection{Numerical simulations}

Bifurcation analysis of the ring of seven identical Duffing oscillators (Eq. (5)) allows one to follow the scenario of transition to chaos in this system. Diagrams of individual node response $x_{j}$, the sum of all oscillators responses $\sum^{N} x_{j}(j=1,2, \ldots, 7)$ and corresponding courses of Lyapunov exponents (LEs), calculated versus coupling strength $\sigma$, are illustrated in Figures 2a-2c, respectively. Eigenvalue approach of the system under consideration, presented in the previous section, shows that the PRW begins to propagate along the ring after the first Hopf bifurcation of the equilibrium (stationary state) at $\sigma_{1}=0.0329$ (see Fig. 2c). However, this solution dominates only in a very narrow $\sigma$-range as it is shown in Figures $2 \mathrm{a}$ and $2 \mathrm{c}$. As a result of a further increase of the coupling parameter, two successive bifurcations of the Hopf-type 


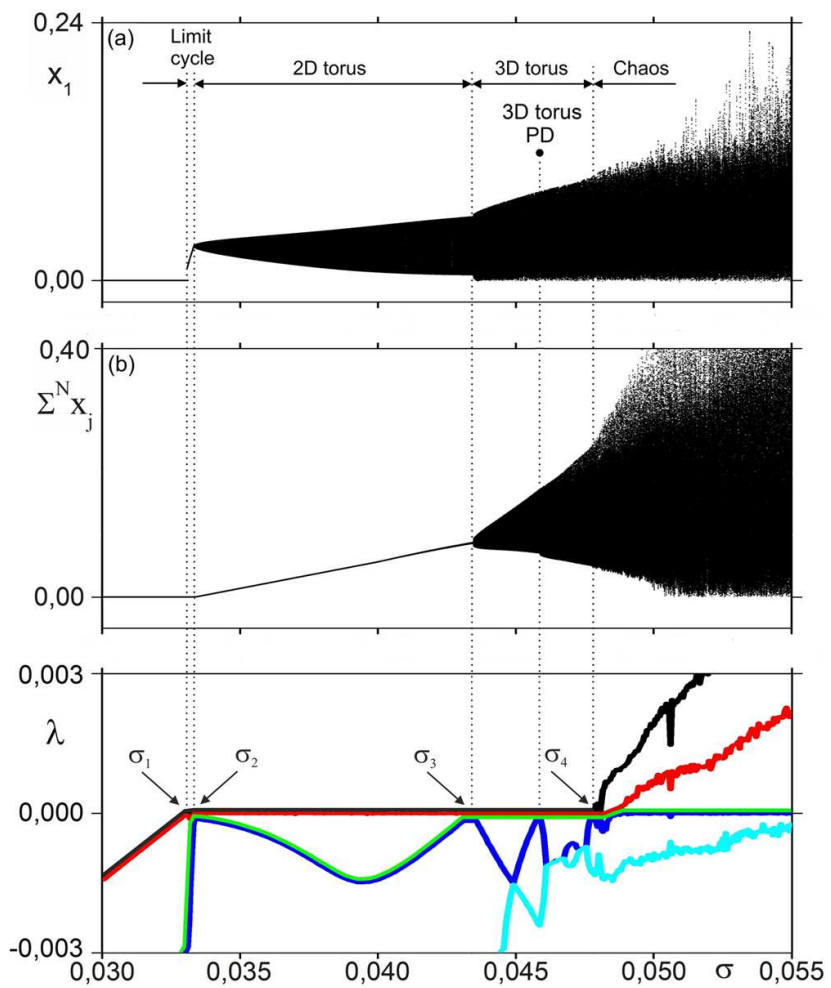

Fig. 2. Bifurcation diagrams of individual node variable $x_{1}$ (a), sum of all variables $\sum^{N} x_{j}(\mathrm{~b})$ and the corresponding five largest LEs (c) for the ring of seven identical Duffing oscillators versus coupling strength $\sigma$. Parameters: $a=1.0 ; b=10.0, d=0.3162$.

lead to the appearance of the $2 \mathrm{D}$ torus (at $\sigma_{2}=0.0333$ ) and then the 3D torus (at $\left.\sigma_{3}=0.0433\right)$. This latter is stable in a relatively wide interval $-\sigma \in[0.0433,0.0478]$. After exceeding the value of $\sigma_{4}=0.0478$ the largest LE becomes positive, so chaotic and hyperchaotic solutions begins to dominate. The hyperchaotic attractor is characterized by the following LEs spectrum: $(+,+, 0,0,-, \ldots,-)$. It means that we observe an unstable $2 \mathrm{D}$ torus embedded inside the hyperchaotic solution. The last Hopf-type bifurcation at $\sigma_{4}$ destabilizes local 2D-torus and thus the $3 \mathrm{D}$ global quasi-periodic solution.

In Figures 3-7 detailed numerical results that illustrate solutions occurring during the transition from periodic (Fig. 3) to chaotic motion (Fig. 7) via a 2D-torus (Fig. 4), a 3D-torus (Fig. 5) and its period doubling (Fig. 6), are demonstrated. Poincare cross-sections of individual oscillators $x_{j}$ (Figs. 3b-7b) are juxtaposed with their time series (Figs. 4a-7a), or all seven nodes courses in Figure 3a, and corresponding time courses of the sum $\sum^{N} x_{j}$ of all signals (Figs. 3d-7d). Additionally, frequency spectra (Figs. 3c-7c), reconstructed from time series and confirming identification of attractors' types, are presented.

In fact, the system under consideration (Eq. (5)) is of seven DoFs (degrees of freedom). Let's treat each of them as a local DoF because they correspond to individual nodes in the loop. However, in the global dynamics of this system an important role is played by rotating wave being an effect of unidirectional local DoFs coupling. Therefore, it can be treated as an additional rotational mode. The extraction of the local responses from global ring can allow one to understand better and identify the mechanism of the $3 \mathrm{D}$ torus stability. This can be achieved by separating the 

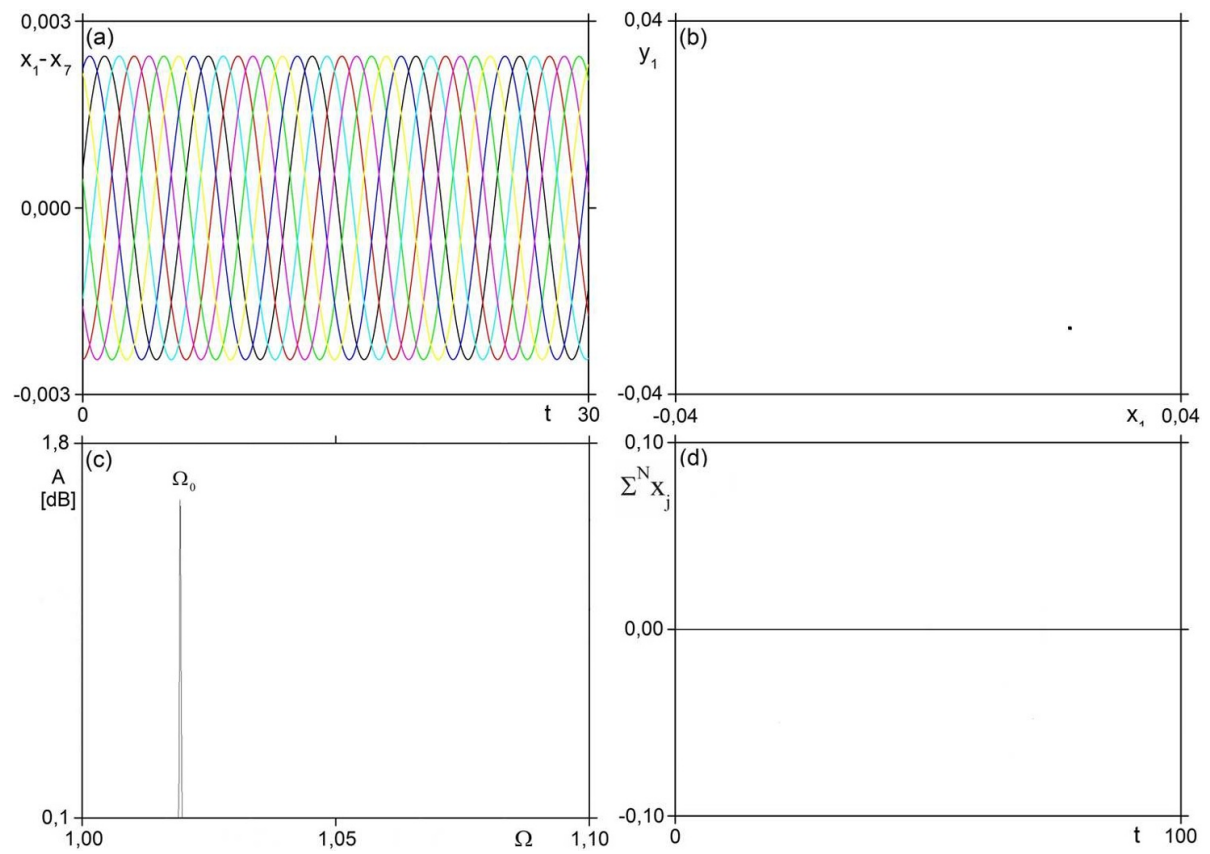

Fig. 3. Time series of all seven oscillators (a), corresponding Poincaré map of the single oscillator (b), the FFT spectrum (c) and time series of the sum of all signals $x_{j}$ (d) illustrating the dynamics of the system (5) for $\sigma=0,0332$.
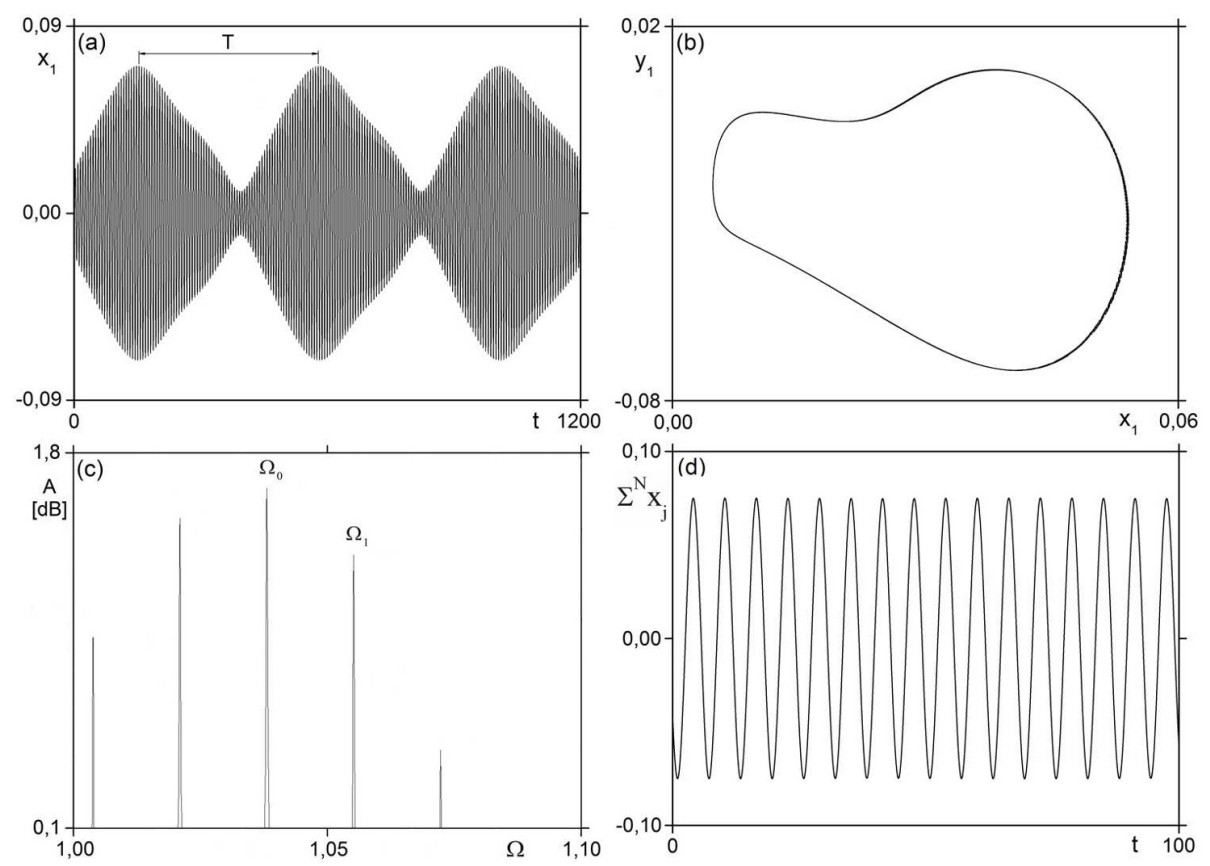

Fig. 4. Time series of the single oscillator (a), corresponding Poincaré map (b), the FFT spectrum (c) and time series of the sum of all signals $x_{j}$ (d) illustrating the dynamics of the system (5) for $\sigma=0,0430$. 

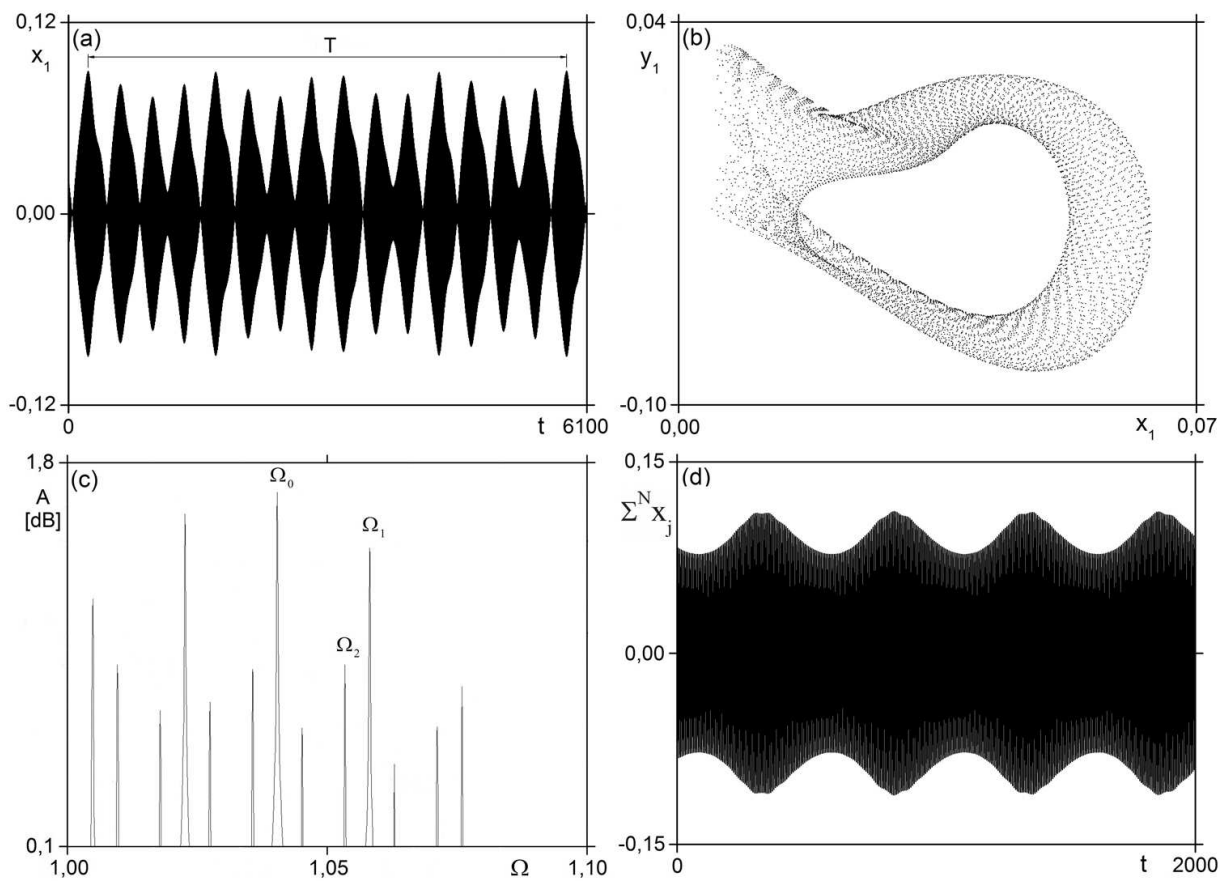

Fig. 5. Time series of the single oscillator (a), corresponding Poincaré map (b), the FFT spectrum (c) and time series of the sum of all signals $x_{j}$ (d) illustrating the dynamics of the system (5) for $\sigma=0,0440$.
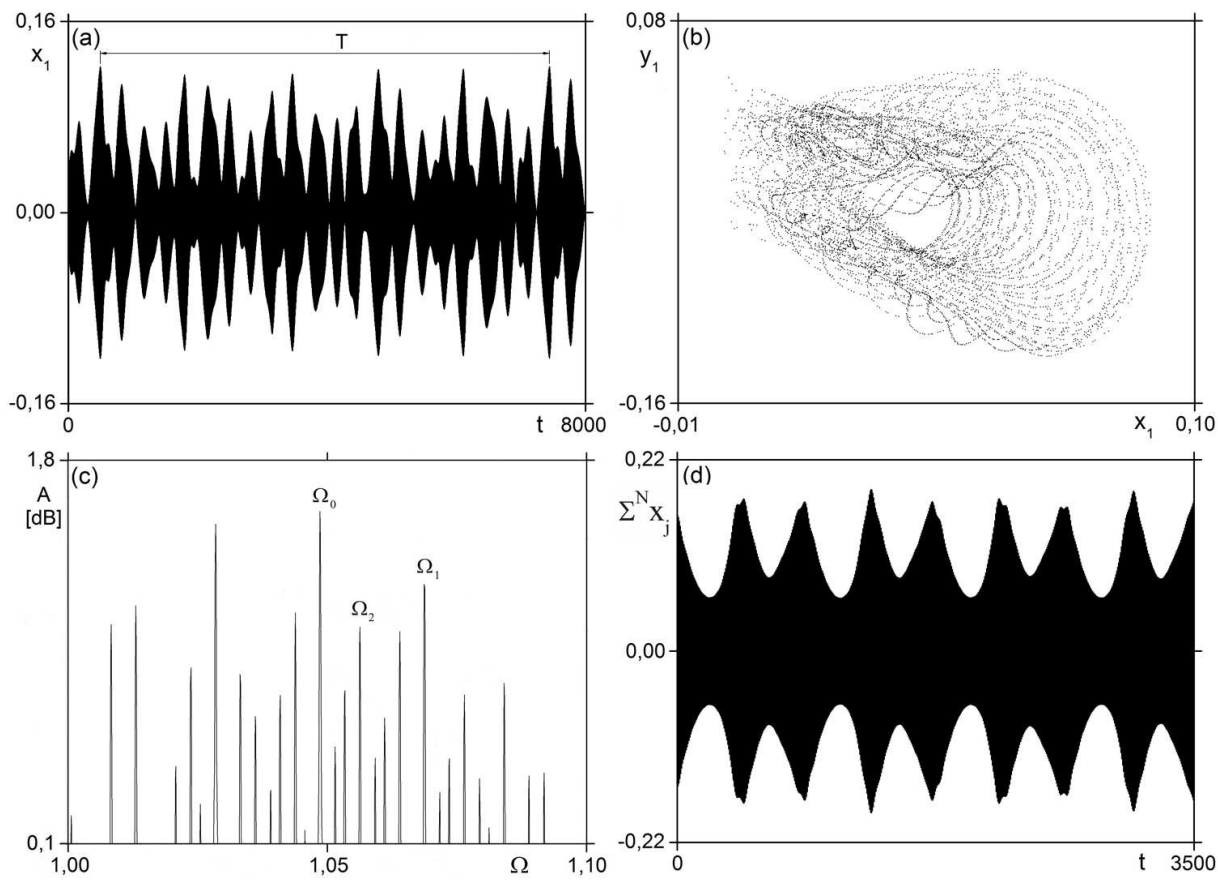

Fig. 6. Time series of the single oscillator (a), corresponding Poincaré map (b), the FFT spectrum (c) and time series of the sum of all signals $x_{j}$ (d) illustrating the dynamics of the system (5) for $\sigma=0,0470$. 

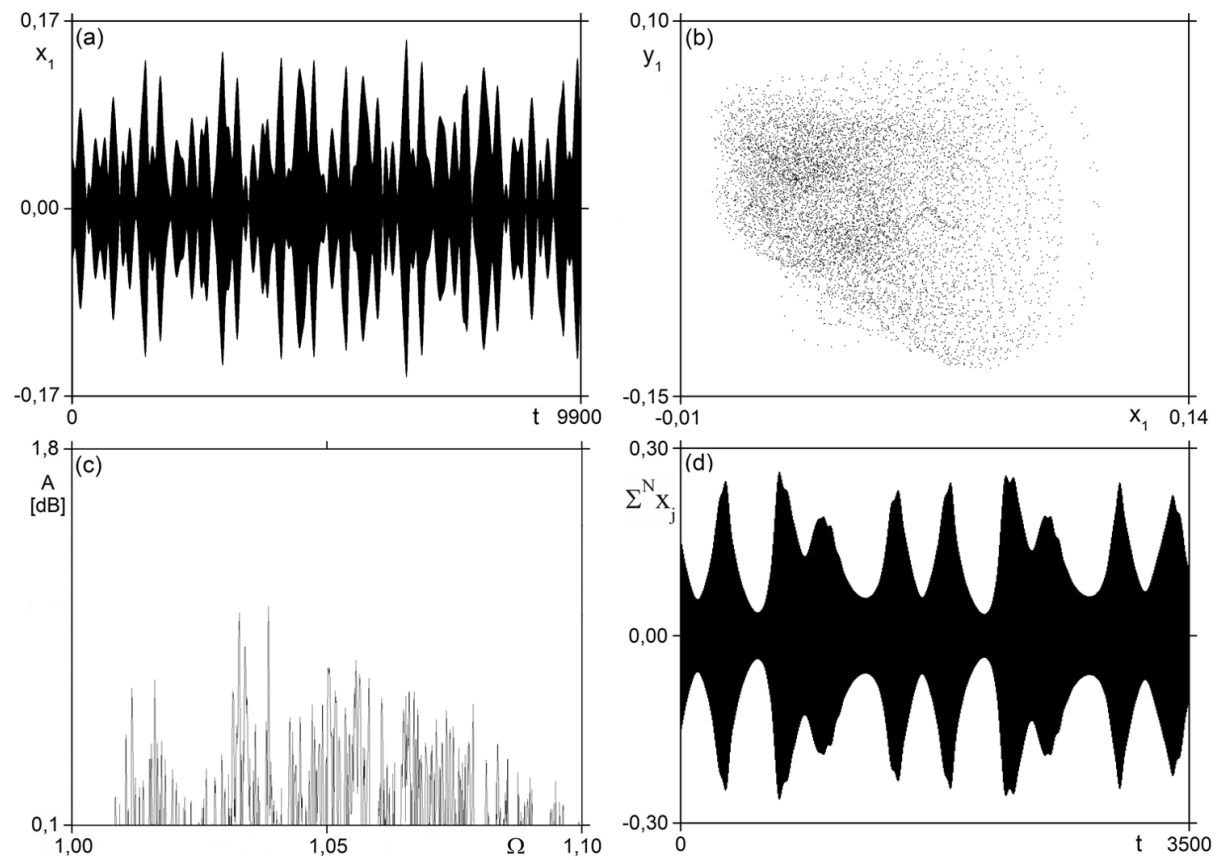

Fig. 7. Time series of the single oscillator (a), corresponding Poincaré map (b), the FFT spectrum (c) and time series of the sum of all signals $x_{j}$ (d) illustrating the dynamics of the system (5) for $\sigma=0,0500$.

rotational forcing, represented with high-frequency PRW components (Fig. 3a), from the registered global ring signal. The same identical periodic components are embedded in the structure, as a skeleton, of quasi-periodic and chaotic solutions. Such PRW modes can be simply eliminated from responses of identical oscillators by summing their signals (Figs. 3d-7d). Due to the symmetry of phase distribution along the ring their sum is equal to zero in each moment of the system evolution (see Fig. 3d). Thus, such sum signal is representative for the local dynamics of ring oscillators. In Figure $2 \mathrm{~b}$ the bifurcation diagram of the sum $\sum^{N} x_{j}$ versus $\sigma$ is demonstrated. This sum remains equal to zero up to a value $\sigma_{2}$, where its Hopf-type bifurcation takes place. Next, the limit cycle of the sum $\sum^{N} x_{j}$ (Fig. $4 \mathrm{~d}$ ), relating to the $2 \mathrm{D}$ torus solution (Figs. 4a and 4b), is observed in the range $\sigma_{2}<\sigma<\sigma_{3}$. The comparison of bifurcation graphs from Figures $2 \mathrm{a}$ and $2 \mathrm{~b}$ in this $\sigma$-range clearly illustrate the extraction effect of the local limit cycle (Fig. 2b) from the global 2D torus (Fig. 2a). Subsequently, the Hopf-type bifurcation at $\sigma_{3}$ leads to the appearance of the local 2D-torus (Figs. 5d and 6d) in the interval $\sigma_{3}<\sigma<\sigma_{4}$, in which a 3D-torus of the global system exists - before (Figs. 5a and 5b) and after (Figs. 6a and 6b) its period doubling at $\sigma=0.0459$. The last Hopf-type bifurcation at $\sigma_{4}$ destabilizes the local $2 \mathrm{D}$-torus and thus the $3 \mathrm{D}$ global quasi-periodic solution. Hence, a chaotic motion becomes dominant (Figs. 7a-7d). Consequently, in the local DoF the classical NRT transition to chaos which is clearly illustrated in Figure 2b, can be observed.

\section{Summary and conclusions}

Analyzing the results presented in Section 3 and taking under consideration hypotheses (mentioned in Sect. 1), formulated by other authors $[17,27,29,40]$, we can propose 

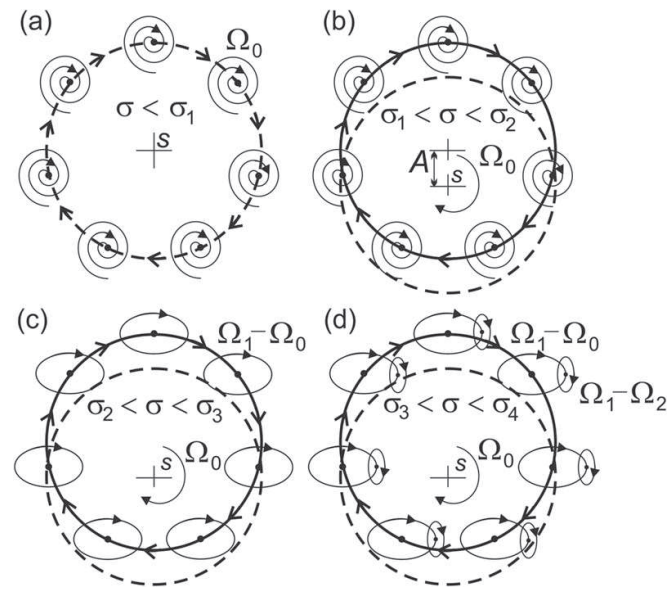

Fig. 8. Pictorial illustration of the conjecture explaining robust stability of the 3D-torus.

a conjecture explaining the observed behavior of the system (1), which is pictorially illustrated in Figures $8 \mathrm{a}-8 \mathrm{~d}$ for the case of identical nodes. According to this approach global, rotational mode, being an effect of unidirectional connectivity scheme, is of different nature than responses of individual nodes in the local DoFs. Therefore, they can be structurally separated. Small coupling $\left(\sigma<\sigma_{1}\right)$ does not enable an initiation of the RW and damped oscillations of each item take place (Fig. 8a), i.e., a global equilibrium position (dotted circle in Fig. 8a) is stable. The Hopf bifurcation at $\sigma_{1}=0.0329$ activates the rotational DoF, i.e. the PRW is propagated along the ring (see Fig. 3b), but local vibrations are still damped. In result we can observe harmonic movement of oscillators with the frequency $\Omega_{0}$ and the amplitude $A$ due to the rotational forcing. On the symbolic plot shown in Figure $8 \mathrm{~b}$ it is manifested by the eccentricity of distance $A$ between the centers of the solid line circle and the dotted line circle. The solid line circle rotating around the center point $S$ symbolizes the rotational mode. Thus, revolutions of this circle demonstrate pictorially the PRW oscillating around the global equilibrium. A small increase in the coupling strength causes a second Hopf type bifurcation at $\sigma_{2}=0.0333$, which is also the first in local DoF. It activates local limit cycles and a new frequency $\Omega_{1}$ (Fig. 8c) which is disproportionate to $\Omega_{0}$ (the PRW frequency). In result, we can see the $2 \mathrm{D}$ torus as a combination of the PRW and the local limit cycle. After the next Hopf-type bifurcation at $\sigma_{3}=0.0433$, a third incommensurate frequency $\Omega_{2}$ appears and a stable 3 -frequency quasi-periodic solution can be observed (see Figs. 5a-5c and $6 \mathrm{a}-6 \mathrm{c}$ ). However, according to our conjecture the third Hopf bifurcation is only the second in the local DoF, and then the 3D torus can be considered as a superposition of the local 2D torus and the PRW (Fig. 8d). Finally, chaos dominates after crossing the limit $\sigma_{4}=0.0478$ (Figs. 2a-2c and 7a-7d). From the viewpoint of overall ring dynamics it looks like transition to chaos after fourth Hopf-type bifurcation but from the local DoF point of view this is a realization of the classical NRT scenario where the chaotic attractor appears as a product of the 3D torus destruction just after third consecutive Hopf bifurcation (compare Figs. 2a and $2 \mathrm{~b}$ ). Thus, the ring dynamics is a superposition of periodic rotational forcing and local responses of individual oscillators. In the case of the $3 \mathrm{D}$ torus such high-frequency forcing (characterized by $\Omega_{0}$ ) coexists with low-frequency local responses represented by dominant frequency differences $\Omega_{1}-\Omega_{0}$ and $\Omega_{1}-\Omega_{2}$ (Fig. 8d). Significant disparity of these frequencies (the PRW and local limit cycles) is illustrated on frequency spectra in Figures 5c and 6c. As we mentioned above, such disparity of the 3D torus frequencies was postulated as the cause of its stability [29]. 
Summing up, we have demonstrated in numerical experiment the way to chaos via four consecutive Hopf-type bifurcation and a stable 3-frequency quasi-periodic solution in the ring of unidirectionally coupled, identical Duffing oscillators. The presented analysis should be considered as a supplement to our previous experimental studies on this research problem [9]. In this paper we have concentrated on the causes and mechanism of the 3D-torus stability. The mechanism of such stability has been explained as an effect of the structural separation of rotational and local DoFs. According to our conjecture, the existence of the stable 3D torus is a result of synchronous correlation of the PRW and the 2-frequency quasiperiodic response of nodal oscillators. Our research (including not yet published results) allows us to suppose that the 3 -frequency quasi-periodicity is a phenomenon more common for loops of unidirectionally coupled oscillators, e.g., the 3D torus observed in the ring of coupled Lorenz systems [27-29]. A verification of this hypothesis is our task for the nearest future.

This study has been supported by the Polish National Centre of Science (NCN) under project No. 2017/27/B/ST8/01619.

Publisher's Note The EPJ Publishers remain neutral with regard to jurisdictional claims in published maps and institutional affiliations.

Open Access This is an open access article distributed under the terms of the Creative Commons Attribution License (http://creativecommons.org/licenses/by/4.0), which permits unrestricted use, distribution, and reproduction in any medium, provided the original work is properly cited.

\section{References}

1. L.D. Landau, Dokl. Akad. Nauk SSSR 44, 339 (1944)

2. E. Hopf, Commun. Pure Appl. Math. 1, 303 (1948)

3. S. Newhouse, D. Ruelle, F. Takens, Commun. Math. Phys. 64, 35 (1978)

4. H.T. Moon, P. Huerre, LG. Redekopp, Phys. Rev. Lett. 49, 458 (1982)

5. R. Alaggio, G. Rega, Physica D 137, 70 (2000)

6. P. Linsay, A. Cummings, Physica D 40, 196 (1989)

7. A. Libchaber, J. Mauer, Proceedings of NATO Advanced Summer Institute on Nonlinear Phenomena, 1982, p. 259

8. S. Martin, H. Leber, W. Martienssen, Phys. Rev. Lett. 49, 303 (1984)

9. L. Borkowski, P. Perlikowski, T. Kapitaniak, A. Stefanski, Phys. Rev. E 91, 062906 (2015)

10. N.V. Stankevich, A.P. Kuznetsov, E.S. Popova, E.P. Seleznev, Commun. Nonlinear Sci. Numer. Simul. 43, 200 (2017)

11. R.V. Buskirk, C. Jeffries, Phys. Rev. A 31, 3332 (1985)

12. C. Baesens, J. Guckenheimer, S. Kim, R.S. MacKay, Physica D 49, 387 (1991)

13. C. Grebogi, E. Ott, J. Yorke, Phys. Rev. Lett. 51, 339 (1983)

14. C. Grebogi, E. Ott, J. Yorke, Physica D 15, 354 (1985)

15. P. Ashwin, J. Guasch, J.M. Phelps, Physica D 66, 392 (1993)

16. P. Ashwin, Chaos Solitons Fractals 9, 1279 (1998)

17. J.J. Collins, I. Stewart, Biol. Cybern. 71, 95 (1994)

18. K. Rompala, R. Rand, H. Howland, Commun. Nonlin. Sci. Num. Simul. 12, 794 (2007)

19. M. Battelino, Phys. Rev. A 38, 1495 (1988)

20. U. Feudel, W. Jansen, J. Kurths, Int. J. Bifurc. Chaos 3, 131 (1993)

21. U. Feudel, M. Safonova, J. Kurths, V. Anishchenko, Int. J. Bifurc. Chaos 6, 1319 (1996)

22. V. Anishchenko, M. Safonova, U. Feudel, J. Kurths, Int. J. Bifurc. Chaos 4, 595 (1994)

23. J. Yang, Phys. Rev. E 61, 6521 (2000) 
24. H.T. Moon, Phys. Rev. Lett. 79, 35 (1997)

25. J.M. Lopez, F. Marques, Phys. Rev. Lett. 85, 972 (2000)

26. F. Marques, J.M. Lopez, J. Shen, Physica D 156, 81 (2001)

27. D. Pazo, E. Sánchez, M.A. Matias, Int. J. Bifurc. Chaos 11, 2683 (2001)

28. D. Pazo, M.A. Matias, Europhys. Lett. 72, 176 (2005)

29. E. Sánchez, D. Pazo, M.A. Matías, Chaos 16, 033122 (2006)

30. G.V. Osipov, J. Kurths, S. Zhou, Synchronization in oscillatory networks (Springer, Berlin, 2007)

31. B. Qinsheng, Int. J. Nonlinear Mech. 39, 33 (2004)

32. W. Wu, Z. Chen, Z. Yuan, Chaos Solitons Fractals 39, 2340 (2009)

33. N.V. Stankevich, J. Kurths, A.P. Kuznetsov, Commun. Nonlinear Sci. Numer. Simul. 20, $316(2015)$

34. V. Anishchenko, S. Nikolaev, Int. J. Bifurc. Chaos 18, 2733 (2008)

35. A.P. Kuznetsov, S.P. Kuznetsov, I.R. Sataev, L.V. Turukina, Phys. Lett. A 377, 3291 (2013)

36. S. Hidaka, N. Inaba, M. Sekikawa, T. Endo, Phys. Lett. A 379, 664 (2015)

37. P. Perlikowski, S. Yanchuk, M. Wolfrum, A. Stefanski, P. Mosiolek, T. Kapitaniak, Chaos 20, 013111 (2010)

38. S. Yanchuk, P. Perlikowski, M. Wolfrum, A. Stefanski, T. Kapitaniak, Chaos 25, 033113 (2015)

39. L. Borkowski, A. Stefanski, Math. Prob. Eng. 2015, 367036 (2015)

40. D. Rand, Arch. Ration. Mech. Anal. 79, 1 (1982)

41. M. Wolfrum, S. Yanchuk, Phys. Rev. Lett. 96, 220201 (2006)

42. S. Yanchuk, M. Wolfrum, Phys. Rev. E 77, 026212 (2008) 